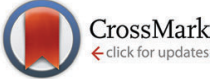

Cite this: Phys. Chem. Chem. Phys., 2016, 18, 22590

Received 11th May 2016, Accepted 22nd July 2016

DOI: $10.1039 / c 6 c p 03182 j$

www.rsc.org/pccp

\section{Breathing mode vibrations and elastic properties of single-crystal and penta-twinned gold nanorods $\uparrow$}

\begin{abstract}
Yong Gan, ${ }^{* a}$ Zheng Sun ${ }^{a}$ and Zhen Chen ${ }^{b}$
The acoustic vibrations of individual single-crystal and penta-twinned gold nanorods with widths from $\sim 7$ to $\sim 26 \mathrm{~nm}$ are studied using atomic-level simulations and finite element calculations. It is demonstrated that the continuum model in the limit of an infinite rod length could be used to describe the breathing periods of nanorods with an aspect ratio as small as $\sim 2.5$, in combination with bulk material elastic constants. The elastic moduli of gold nanorods are determined via their atomistically simulated extensional periods and the dispersion relation based on long-wavelength approximation. The twinned nanorods become stiffer as the width is reduced, which is in contrast to the size dependence of the modulus in single-crystal nanorods. Further finite element calculations for the breathing periods of nanorods are performed using isotropic elastic constants of bulk gold. We find that the breathing vibrations of the penta-twinned nanorods are more affected by the crystal structure effect than those of single-crystal nanorods, because a smaller range of crystal directions perpendicular to the long axis is involved in the breathing vibrations of twinned nanorods.
\end{abstract}

\section{Introduction}

Since their first synthesis in the $1990 \mathrm{~s},{ }^{1,2}$ the fabrication capabilities of gold nanorods have been significantly enhanced in terms of the yield and quality of nanorods, ${ }^{3-5}$ promoting numerous efforts devoted to exploring their optical, electronic and mechanical properties. ${ }^{5-7}$ Acoustic vibrations of gold nanorods have been the subject of an increasing amount of research. ${ }^{8-10}$ This is mainly motivated by the promising potential of vibrating nanorods in the applications of ultrasensitive sensing and nano-optics. ${ }^{11-13}$ Moreover, vibrational responses of gold nanorods provide accurate information of their mechanical properties, ${ }^{8,10,14}$ which is of crucial importance, both from a fundamental point of view to understand the effects of sizes and crystal structures on the mechanical properties of nanoobjects, and from a practical viewpoint to design gold nanorodbased sensors and devices.

The optical method based on ultrafast time-resolved spectroscopy has been widely used to study the vibrations of gold nanorods. ${ }^{14-16}$ Many studies have been focused on the measurements of the vibrational responses of ensembles of gold nanorods. ${ }^{16-20}$

\footnotetext{
${ }^{a}$ Faculty of Engineering, Zhejiang University, Hangzhou, Zhejiang 310027, China. E-mail: ganyong@zju.edu.cn

${ }^{b}$ Department of Civil \& Environmental Engineering, University of Missouri, Columbia, MO 65211, USA

$\dagger$ Electronic supplementary information (ESI) available. See DOI: 10.1039/c6cp03182j
}

However, ensemble measurements provide the average responses of nanorods, and thus obscure a deeper perspective on the sizeand shape-dependent mechanical properties of gold nanorods due to the inevitable inhomogeneity in the size and shape of samples. To overcome this limitation in ensemble measurements, a growing number of time-resolved spectroscopic studies have been conducted on single gold nanorods. ${ }^{21-24}$ Although singleparticle measurements could effectively eliminate the problems resulting from the sample heterogeneity, they suffer from a weaker signal-to-noise ratio as compared with ensemble studies. Thus, it is a challenging task to measure the vibrational responses of single gold nanorods with a small width (e.g., less than $30 \mathrm{~nm}$ ). Recently, atomistic modeling has been used to study the laserexcited extensional vibrations of individual gold nanorods with widths from $\sim 7$ to $\sim 24 \mathrm{~nm}$, and shown to be a powerful means complementary to time-resolved spectroscopy for investigating the vibrations of small single nanorods. ${ }^{25,26}$

So far, the extensional vibration of gold nanorods has been well studied and understood, while there are rare reports on the size and crystal structure dependence of the breathing vibration of gold nanorods. In addition, although the elastic properties of gold nanorods have been intensively investigated, most studies have concentrated on single-crystal nanorods, and there is a lack of a systematic investigation for penta-twinned nanorods. Hence, our understanding is limited with respect to the impact of the crystal structure on the mechanical properties of gold nanorods, especially for small gold nanorods of less than $30 \mathrm{~nm}$ width. 
Here we study the ultrafast laser-excited breathing vibrations and the elastic properties of different-sized individual singlecrystal and penta-twinned gold nanorods, by means of atomistic simulations and finite element (FE) calculations. It is shown that the breathing periods of gold nanorods with an aspect ratio down to $\sim 2.5$ could be well predicted by the continuum elastic model in the limit of an infinitely long rod, with the use of elastic constants of bulk gold. Furthermore, we determine the Young's modulus of penta-twinned gold nanorods through their extensional periods, in combination with a continuum mechanics model, and find that the Young's modulus of pentatwinned nanorods increases as the rod width decreases, which is contrary to the size dependence of elastic modulus of single-crystal nanorods. The FE calculations with isotropic and anisotropic bulk material properties reveal that the breathing vibrations of penta-twinned gold nanorods are more impacted by the crystal structure.

\section{Simulation method}

The acoustic vibrations of free single gold nanorods in a vacuum induced by femtosecond laser irradiation are considered in this study. By following the previous work, ${ }^{27-29}$ the ultrafast laser excitation of gold nanorods is simulated using a hybrid method that couples the two-temperature model (TTM) ${ }^{30}$ into the molecular dynamics (MD) with neglecting energy loss to the surrounding environment and assuming homogeneous laser heating of nanorods. Here we give a brief description of the integrated TTM-MD method, in which the governing equations are ${ }^{27,29}$

$$
\begin{gathered}
C_{\mathrm{e}}\left(T_{\mathrm{e}}\right) \frac{\partial T_{\mathrm{e}}}{\partial t}=-G\left(T_{\mathrm{e}}-T_{\mathrm{l}}\right)+S \\
m_{i} \frac{\mathrm{d}^{2} \boldsymbol{r}_{i}}{\mathrm{~d} t^{2}}=\boldsymbol{F}_{i}-\xi m_{i} v_{i}^{\mathrm{T}}
\end{gathered}
$$

where $C$ is the heat capacity, $T$ is the temperature, $t$ is the time, $G$ is the electron-lattice coupling parameter, $S$ is the volumetric absorbed laser energy per unit time, $m_{i}, \boldsymbol{r}_{i}$ and $v_{i}^{\mathrm{T}}$ are the mass, position and thermal velocity of atom $i$, respectively, $\boldsymbol{F}_{i}$ is the total interatomic forces acting on atom $i$ with the subscript $i$ looping over all atoms, $\xi$ is a variable characterizing the electron-lattice energy exchange, and the subscripts e and l denote the quantities for the electrons and the lattice, respectively.

The laser energy source $S$ is expressed as a temporal Gaussian profile $^{28,29}$

$$
S=0.94 \frac{J_{\mathrm{abs}}}{t_{\mathrm{p}}} \exp \left[-2.77\left(\frac{t-2 t_{\mathrm{p}}}{t_{\mathrm{p}}}\right)^{2}\right]
$$

with $J_{\text {abs }}$ denoting the volumetric absorbed energy by the nanorod, and $t_{\mathrm{p}}$ being the full width at half maximum duration of a laser pulse. The explicit finite difference (FD) scheme is used to solve eqn (1) for the electron temperature. In each MD integration step, an integer number of $n$ FD iterations are performed. Thus, the variable $\xi$ is calculated by ${ }^{27}$

$$
\xi=\frac{\frac{1}{n} V_{\mathrm{NR}} \sum_{j=1}^{n} G\left(T_{1}-T_{\mathrm{e}}^{j}\right)}{\sum_{i=1}^{N_{\mathrm{NR}}} m_{i}\left(\boldsymbol{v}_{i}^{\mathrm{T}}\right)^{2}}
$$

where $T_{\mathrm{e}}^{j}$ represents the electron temperature at the $j$-th FD iteration, and $V_{\mathrm{NR}}$ and $N_{\mathrm{NR}}$ are the volume and total atom number of the nanorod, respectively. In this work, the FD and MD time steps are 0.005 and $2.5 \mathrm{fs}$, respectively, leading to $n=500$ FD iterations within one MD step.

\section{Results and discussion}

The vibrations of both the single-crystal and penta-twinned gold nanorods are taken into account so that the crystal structure effect can be also examined. The all-atom MD models for gold nanorods are created according to the experimentally analyzed crystal structures, ${ }^{31-33}$ as schematically illustrated in Fig. 1. For single-crystal nanorods, four $\{100\}$ and four $\{110\}$ side facets are
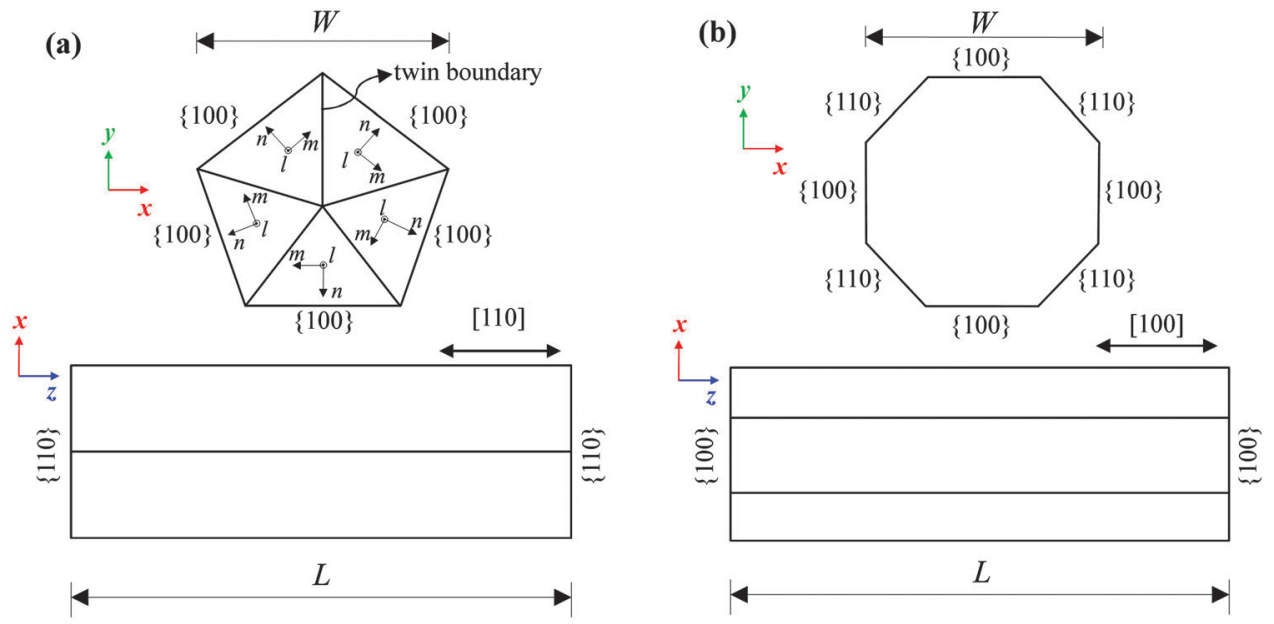

Fig. 1 Schematic illustrations for the shapes and crystal structures of gold nanorods: (a) penta-twinned nanorods with a [100] growth direction. The nanorods are constructed from five single-crystal triangular prisms radially arranged along a common [110] axis. In each of them, the local $l-, m-a n d$ $n$-axes are along the [110], [-110] and [001] crystallographic directions, respectively. (b) Single-crystal nanorod with a [100] growth direction. 
Table 1 Dimensions of simulated gold nanorods. The length $L$ and width $W$ are the average values during the time period 20 ps after laser irradiation. The samples are labeled with $\mathrm{S}$ and $\mathrm{P}$ denoting single-crystal and penta-twinned structures, respectively

\begin{tabular}{llrl}
\hline Sample & $L(\mathrm{~nm})$ & $W(\mathrm{~nm})$ & Aspect ratio $(L / W)$ \\
\hline S1 & 24.57 & 7.15 & 3.43 \\
S2 & 42.94 & 12.44 & 3.45 \\
S3 & 57.22 & 16.52 & 3.46 \\
S4 & 71.44 & 20.63 & 3.46 \\
S5 & 85.73 & 24.70 & 3.47 \\
S6 & 17.70 & 7.15 & 2.48 \\
S7 & 40.91 & 16.52 & 2.48 \\
S8 & 20.98 & 7.16 & 2.93 \\
S9 & 49.32 & 16.63 & 2.97 \\
T1 & 26.25 & 7.54 & 3.48 \\
T2 & 39.58 & 11.30 & 3.50 \\
T3 & 52.90 & 15.09 & 3.50 \\
T4 & 69.56 & 19.78 & 3.52 \\
T5 & 79.47 & 22.68 & 3.50 \\
T6 & 92.73 & 26.47 & 3.50 \\
T7 & 19.05 & 7.53 & 2.53 \\
T8 & 38.21 & 15.06 & 2.54 \\
T9 & 22.79 & 7.54 & 3.02 \\
T10 & 45.69 & 15.06 & 3.03 \\
\hline
\end{tabular}

parallel to the [100] growth direction, and two $\{001\}$ facets are located at the ends. Penta-twinned nanorods with a [110] growth direction have five $\{100\}$ side facets and two $\{110\}$ end facets. In all nanorod models, the side facets have an approximately equal area. The $z$-axis is set along the rod axis and the $x$ - and $y$-axes are positioned in the cross-section plane, as shown in Fig. 1 . The vibrations of totally 19 nanorods, with their detailed dimensions as given in Table 1 , are investigated.

The interatomic interactions between gold atoms are described using the embedded atom method (EAM) potential formulated in ref. 34. This EAM potential predicts the elastic constants of $c_{11}=204.7 \mathrm{GPa}, c_{12}=170.66 \mathrm{GPa}$ and $c_{44}=43.39 \mathrm{GPa}$ for bulk single-crystal gold at $298 \mathrm{~K},{ }^{35}$ which are in good agreement with the experimental values of $c_{11}=192.34 \mathrm{GPa}, c_{12}=163.14 \mathrm{GPa}$ and $c_{44}=41.95 \mathrm{GPa}^{36}$ The thermophysical parameters of $C_{\mathrm{e}}$ and $G$ for gold in ref. 37 are used and the free surface conditions are applied in all directions. Once the nanorod models are built, they are equilibrated for $2 \mathrm{~ns}$ in the $N V T$ ensemble using the Nosé-Hoover thermostat. ${ }^{38,39}$ The equilibrated temperature is set at $50 \mathrm{~K}$ to achieve a high signal-tonoise level for the vibration responses at $\sim 298 \mathrm{~K}$. Thereafter, the 100 -fs laser irradiation of nanorods is simulated in the $N V E$ ensemble. As suggested in ref. 25 and 26, specific values of $J_{\mathrm{abs}}$ corresponding to the absorbed molar energy of $\sim 6.3 \mathrm{~kJ} \mathrm{~mol}^{-1}$ are adopted for attaining a final equilibrium temperature at $\sim 298 \mathrm{~K}$.

In addition to the modulations in optical responses, the laser-excited vibrations of gold nanorods also result in the oscillations of local displacements and internal pressure as well as the volume changes. Existing experiments generally measured the optical responses of nanorods to identify their vibration periods and damping. ${ }^{9,10,16}$ It seems that the vibrations of gold nanorods could be investigated via their volume changes. For atomistic nanorods in vibration, however, it is not easy to accurately compute their volumes. Furthermore, at $\sim 298 \mathrm{~K}$, the thermal oscillations of atoms could lead to a significantly lower signal-to-noise ratio for the volume changes of nanorods as compared with that for the dynamic pressures of nanorods. Alternatively, we study the vibrational responses of nanorods through the internal pressure-related quantities $P_{\alpha \beta}$, defined as follows: ${ }^{40}$

$$
P_{\alpha \beta}=\sum_{i=1}^{N_{\mathrm{NR}}}\left(\frac{v_{i \alpha} v_{i \beta}}{m_{i}}+\frac{1}{2} \sum_{j=1}^{N_{i}} r_{i j \alpha} f_{i j \beta}\right)
$$

where $\alpha, \beta=x, y, z ; v_{i \alpha}$ and $v_{i \beta}$ are the momentum components of atom $i$ in the $\alpha$ and $\beta$ directions, respectively; $r_{i j \alpha}$ is the component of the position vector between atoms $i$ and $j$ in the $\alpha$ direction; $f_{i j \beta}$ is the component of the interatomic force vector on atom $i$ due to atom $j$ in the $\beta$ direction; $N_{i}$ is the total number of the neighboring atoms of atom $i$ within the distance cutoff. Note that the oscillations of lateral $P_{x x}$ and $P_{y y}$ in nanorods are mainly contributed by the breathing vibration of nanorods. The breathing periods of single nanorods are thus obtained via the spectral analysis of $P_{\mathrm{s}}=\left(P_{x x}+P_{y y}\right) / 2$.

We firstly focus on the breathing mode of single-crystal nanorods S1-S5. Fig. 2a and b present the time-histories and the power spectra of $P_{\mathrm{S}}$ in nanorods $\mathrm{S} 1$ and $\mathrm{S} 3$, respectively. The dominant peaks with a high amplitude in the power spectra are assigned to the fundamental breathing mode of gold nanorods, as discussed below.

By the FE calculations, the fundamental breathing mode of the octagonal cross-section of single-crystal nanorods characterized by the motion at the vertices is obtained, as shown in Fig. 2c. The cross-sectional dimensions of nanorods in the $\mathrm{FE}$ calculations follow those listed in Table 1 and the plain strain assumption is applied. Moreover, the FE calculations are conducted using the material properties for bulk fcc-crystal gold, including the density of $19300 \mathrm{~kg} \mathrm{~m}^{-3}$ and the anisotropic elastic constants of $c_{11}=204.7 \mathrm{GPa}, c_{12}=170.66 \mathrm{GPa}$ and $c_{44}=43.39 \mathrm{GPa}$ predicted by the adopted EAM potential. As demonstrated in Fig. 2d, the FE-calculated breathing periods of nanorods agree well with the periods corresponding to the peaks in the power spectrum of $P_{\mathrm{s}}$.

Fig. $2 \mathrm{c}$ also presents the top-view atomic pictures of half-cut nanorods S3 at time $t=22.5 \mathrm{ps}$ with the atoms colored by their values of $d_{\mathrm{s}}=\sqrt{d_{x}^{2}+d_{y}^{2}}$, where $d_{x}$ and $d_{y}$ denote the atomic displacements along the $x$ - and $y$-axes, respectively, and are computed by the subtraction between the initial and current atomic coordinates. It is evidently seen that the distributions of $d_{\mathrm{s}}$ by the atomistic simulations are consistent with the FE-calculated fundamental breathing mode corresponding to the vertex motion. Therefore, not only is the assignment of dominant peaks in the power spectra to the fundamental breathing mode confirmed, but also the use of bulk elastic constants for the breathing mode of single-crystal gold nanorods is validated. Moreover, a lower-frequency peak with much lower amplitude appears in the power spectrum of the smaller sample S1. It actually corresponds to the fundamental extensional mode of the nanorod. 

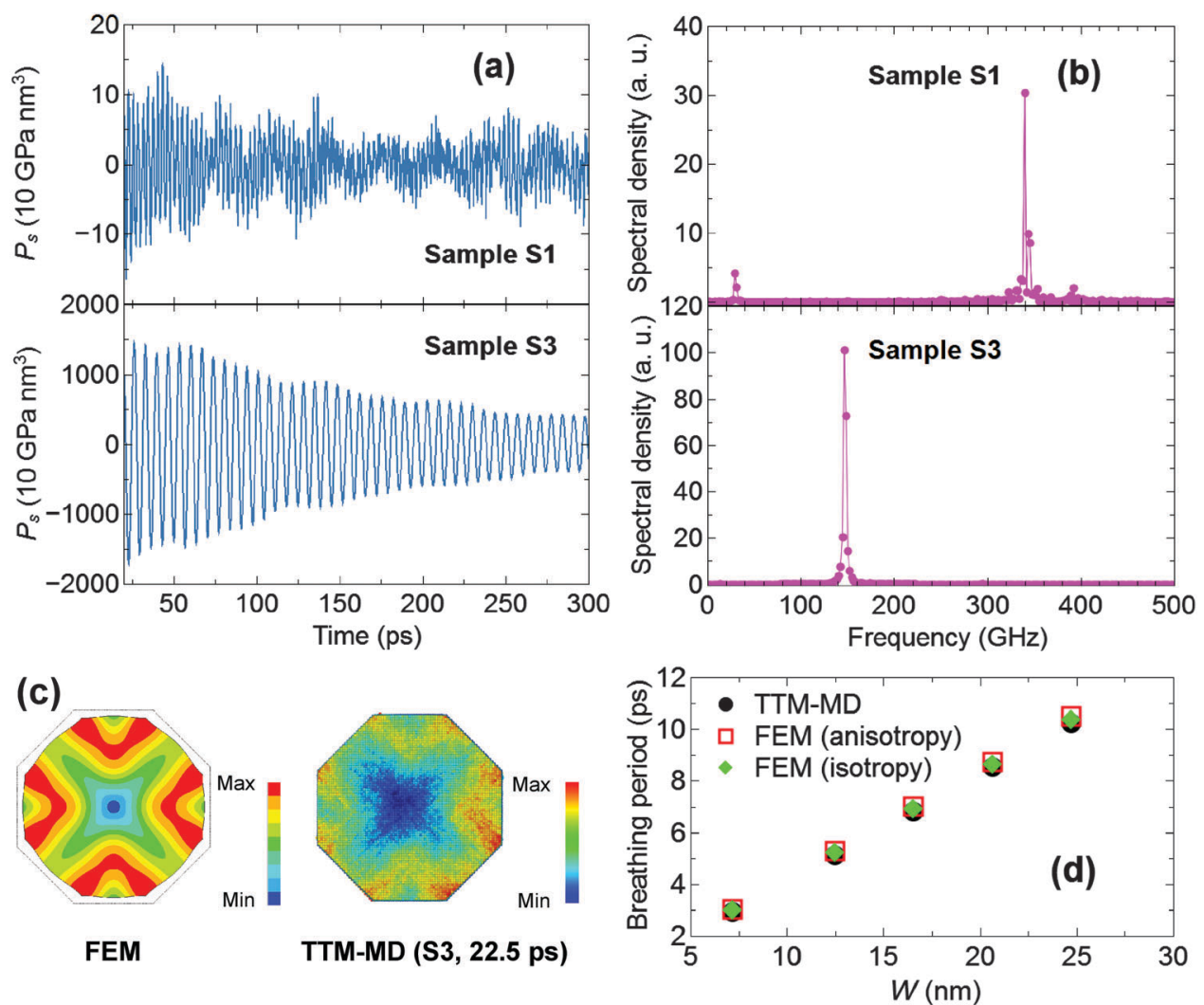

Fig. 2 (a) Transient $P_{\mathrm{s}}$ in single-crystal nanorods S1 and S3. (b) The power spectrum of $P_{\mathrm{s}}$ in samples S1 and S3. (c) FE-calculated fundamental breathing mode with vertex motion and the top-view atomic snapshot of half-cut nanorod S3 at $22.5 \mathrm{ps}$. The atoms in the snapshot are colored according to $d_{\mathrm{s}}$. (d) Comparisons of breathing periods of nanorods S1-S5 obtained using the FE and atomistic simulations. The anisotropic and isotropic elastic constants of bulk gold obtained by the adopted EAM potential are both used in the FE calculations.

The breathing vibrations of penta-twinned nanorods T1-T6 are further analyzed using the same methodology and procedure. The transient $P_{\mathrm{s}}$ in twinned nanorods T1 and T3 and the corresponding power spectra are separately shown in Fig. $3 \mathrm{a}$ and $\mathrm{b}$. Again, the lower-frequency peak for the fundamental extensional mode appears in the power spectrum of the smaller twinned nanorod T1. As can be seen from Fig. 3b, two distinct breathing modes of penta-twinned nanorods with close frequencies are excited. The lower-frequency mode corresponds to the motion at the vertices, while the higher-frequency mode is associated with the motion at the edges of the pentagonal cross-section. These attributions can be proved by Fig. $3 \mathrm{c}$ and d, which compare the cross-sectional motions and vibrational periods by the FE and atomistic simulations, respectively.

It is shown in Fig. 3c and d that, in addition to a good agreement in the breathing periods, the atomic displacement patterns in the cross-sectional plane reasonably match two breathing modes with the motions at the vertices and edges of the pentagon as revealed by the FE study. Based on the scheme in Fig. 1a, the FE models for the cross-sections of twinned nanorods are constructed by five triangles with the same material properties. For each triangle, the anisotropic elastic constants in the local $l-m-n$ coordinate system are derived from $c_{11}, c_{12}$, and $c_{44}$ of bulk fcc-crystal gold by the tensor transformation ${ }^{41}$ and used in the $\mathrm{FE}$ calculations. It is worth mentioning that these two breathing modes have also been observed in a recent experiment on the vibrations of single gold nanorods with an average width of $\sim 45 \mathrm{~nm}^{24}$ In view of the results in Fig. 2 and 3, it is pointed out that the breathing periods of single gold nanorods in either the single-crystal or penta-twinned structure could be well described using the macroscopic elastic model, together with the bulk material elastic constants.

The elastic moduli of penta-twinned gold nanorods are studied next, for which the fundamental extensional periods of gold nanorods $T_{\text {ext }}$ are required. Basically, the fundamental extensional periods of nanorods can be found through the spectral analysis of $P_{z z}$ or the quantities containing $P_{z z}$. However, the frequency resolution of spectral analysis might not be high enough to guarantee the accuracy of extensional periods, especially for long nanorods. Therefore, the extensional periods of all gold nanorods are obtained by fitting the transient $z$-axial displacement of the center-of-mass (COM) in a $1.2 \mathrm{~nm}$-thick slab at the sample end, $d_{\mathrm{COM}}$, to a cosine function, as proposed in our previous work. ${ }^{26}$

Fig. 4a shows the cosine curves fitted to the transient $d_{\mathrm{COM}}$ data for samples T1 and T3 at the time 20 ps after the laser irradiation, which are calculated by subtracting the averaged $z$-coordinate of the slab COM over the simulation time period from the transient $z$-coordinate. It has been proved that the dispersion relation based on the long-wavelength limit is valid 

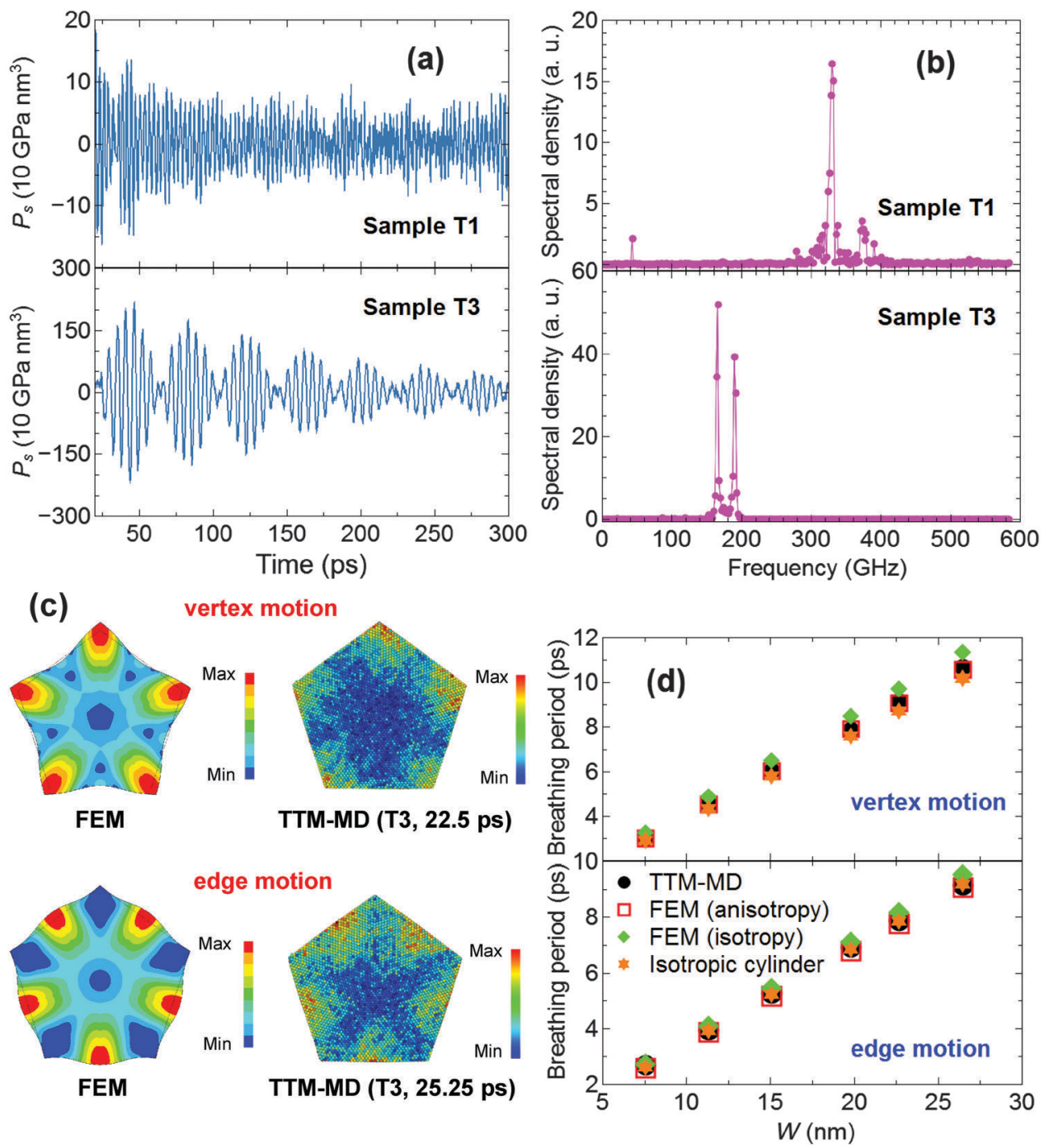

Fig. 3 (a) Time-histories of $P_{\mathrm{s}}$ in penta-twinned nanorods T1 and T3. (b) The power spectrum of $P_{\mathrm{s}}$ in samples T1 and T3. (c) FE-calculated fundamental breathing modes corresponding to motion at the vertices and the edges of the pentagon and the top-view atomic snapshots of the half-cut nanorod T3 at 22.5 and $25.25 \mathrm{ps}$. The atoms are colored based on $d_{\mathrm{s}}$. (d) Comparisons of breathing periods of nanorods T1-T6 obtained by the FE and atomistic analysis. The anisotropic and isotropic elastic constants of bulk gold predicted by the EAM potential are both used in the FE calculations. The star symbols in (d) represent the periods for the fundamental breathing mode (the edge motion panel) and the five-fold surface mode (the vertex motion panel) of the isotropic elastic cylinder with the same radius as that for the incircle of the pentagon cross-section of twinned nanorods.

for the fundamental extensional vibration of metallic nanorods with an aspect ratio as small as $\sim 2.4 .^{10,17,26,42}$ As a result, we determine the elastic moduli of gold nanorods $E_{\mathrm{NR}}$ using the dispersion relation of $T_{\mathrm{ext}}=2 L / \sqrt{E_{\mathrm{NR}} / \rho}$, with density $\rho=19300 \mathrm{~kg} \mathrm{~m}^{-3}$, length $L$ in Table 1 , and extensional period $T_{\text {ext }}$ derived by fitting $d_{\text {COM}}$. Fig. $4 \mathrm{~b}$ displays the relationship between the modulus $E_{\mathrm{NR}}$ and the rod width $W$ for samples T1-T6. The dependence of the Young's moduli of nanorods S1-S5 on their width is also included in Fig. 4 b, for showing the crystal structure effect on the mechanical properties of gold nanorods. In contrast to the size effect in single-crystal nanorods, the penta-twinned gold nanorods become stiffer with the decrease of the width. For example, the Young's moduli of the twinned nanorods $\mathrm{T} 1(W=\sim 7.54 \mathrm{~nm}), \mathrm{T} 2(W=\sim 11.3 \mathrm{~nm})$ and $\mathrm{T} 3(W=\sim 15.1 \mathrm{~nm})$ are $\sim 11 \%, \sim 8 \%$ and $\sim 6 \%$ larger than the modulus of bulk gold in the [110] direction $E_{[110]}$, respectively. Indeed, the hardening with the decreasing width is also suggested in the atomistic simulations of penta-twinned metallic nanowires under tension. ${ }^{43}$ With the increasing $W$, the elastic modulus of twinned nanorods is closer to the bulk value $E_{[110]}$.

The stiffening or softening of gold nanorods as shown in Fig. 4 could be, in general, attributed to the constraints by the five-fold twinned structure and/or the nonlinear elasticity of the nanorod core due to surface stress. It has been shown that the nonlinear core elasticity predominantly accounts for the decreasing or increasing Young's modulus of gold nanorods with the decrease of their width, depending on the orientation of nanorods. ${ }^{44,45}$ Specifically, the softening is exhibited for the $\langle 100\rangle$ oriented nanorods whereas the $\langle 110\rangle$ oriented nanorods stiffens. Therefore, the softening of single-crystal gold nanorods 

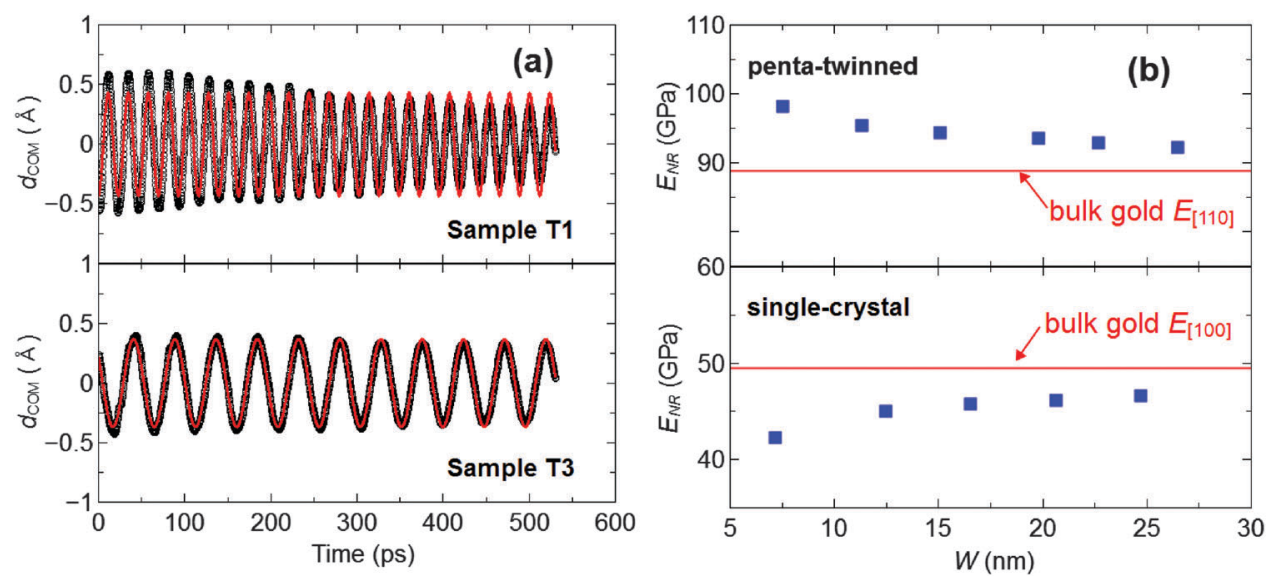

Fig. 4 (a) Transient $z$-axial displacement of the slab COM $d_{\text {COM }}$ for samples T1 and T3. The black circles are the data of $d_{\text {COM }}$ by the combined TTM-MD simulations, and the red curves are the cosine fits to the simulated $d_{\mathrm{COM}}$. (b) Relationships between the Young's moduli of penta-twinned and singlecrystal gold nanorods and their widths. The red lines indicate the Young's moduli of bulk gold along the growth direction of nanorods that are calculated using the EAM potential in the atomistic simulations. The aspect ratios of all nanorods are $\sim 3.5$ (see Table 1).

([100] orientation) mainly results from the nonlinear elasticity within the nanorod cores. For the twinned gold nanorods ([110] orientation), in addition to the nonlinear core elasticity influence, the constraints due to the twinned structure also contribute to their stiffening. ${ }^{43}$ On the other hand, the dispersion relation indicates that the longitudinal wave velocity is proportional to the square root of Young's modulus. The dependence of the atomistically simulated extensional periods of nanorods on their width actually reflects the size-dependent Young's modulus of nanorods due to the constraints by the twinned structure and/or the nonlinear core elasticity, and does not contradict the dispersion relation by the long-wavelength approximation. Furthermore, by the FE calculations (see the ESI $\dagger$ ), we find that the dispersion relation based on the long-wavelength limit could be used for the twinned nanorods with an aspect ratio as small as 2.5. The aspect ratios of nanorods in Fig. $4 \mathrm{~b}$ are all $\sim 3.5$. Thus, a correction analogous to the Rayleigh correction is not required for the dispersion relation.

By fitting the simulated transient $P=\left(P_{x x}+P_{y y}+P_{z z}\right) / 3$ to a sum of damped cosine functions (see the ESI $\dagger$ ), we have determined the damping time $\tau$ of the vibrational motions for nanorods S1-S5 and T1-T6. Tables 2 and 3 give the corresponding intrinsic quality factors $Q_{\text {int }}$ calculated by $Q_{\text {int }}=\pi \tau / T$ with $T$ being the vibrational period. Except $Q_{\text {int }}$ for the extensional mode of twinned nanorods, the other intrinsic quality factors of single-crystal and twinned nanorods are close, and favorably agree with the measured values of $90 \pm 29 .^{46}$ This implies the insignificant crystal-structure effect

Table 2 Intrinsic quality factors $\left(Q_{\text {int }}\right)$ for single-crystal gold nanorods S1-S5. The error quoted for the average $Q_{\text {int }}$ is the standard error

\begin{tabular}{lll}
\hline Sample \# & Extensional mode & Breathing mode \\
\hline S1 & 65.2 & 114.1 \\
S2 & 74.4 & 92.6 \\
S3 & 98.6 & 89.6 \\
S4 & 115.3 & 82.7 \\
S5 & 98.7 & 102.5 \\
Average & $90.4 \pm 9$ & $96.3 \pm 5.5$
\end{tabular}

Table 3 Intrinsic quality factors $\left(Q_{\text {int }}\right)$ for penta-twinned gold nanorods T1-T6. The error quoted for the average $Q_{\text {int }}$ is the standard error

\begin{tabular}{llll}
\hline & & \multicolumn{2}{l}{ Breathing mode } \\
\cline { 3 - 4 } Sample \# & Extensional mode & Vertex motion & Edge motion \\
\hline T1 & 114.4 & 125.9 & 31.4 \\
T2 & 147.7 & 81.5 & 86.8 \\
T3 & 167.5 & 76.0 & 86.7 \\
T4 & 237.9 & 96.6 & 103.4 \\
T5 & 230.3 & 83.0 & 108.4 \\
T6 & 112.8 & 79.2 & 111.7 \\
Average & $168.4 \pm 22.4$ & $90.4 \pm 7.7$ & $88 \pm 12.1$
\end{tabular}

on the damping for the breathing mode of gold nanorods. The larger $Q_{\text {int }}$ for the extensional mode of twinned nanorods is possibly due to the constraints by the five-fold twinned structure. It is worth mentioning that large rod-to-rod variations in the calculated $Q_{\text {int }}$ are observed in Tables 2 and 3, and that a single simulation run has been performed for each sample in this work. However, the varying distributions of initial atomic velocities could lead to the changes in the transient pressures of nanorods, which, in turn, would cause the non-negligible variations in the fitted damping times of nanorods. It is suggested that future efforts be made to perform the combined TTM-MD simulations based on different distributions of initial atomic velocities for each nanorod, toward an improved quantitative analysis of the damping of gold nanorods.

The plane-strain continuum model in our FE analysis strictly holds in the limit of the infinite aspect ratio. The good agreements of breathing periods in Fig. $2 \mathrm{~d}$ and $3 \mathrm{~d}$ imply that this model could also be applicable to gold nanorods of finite aspect ratios. The breathing periods of single-crystal (S6-S9) and penta-twinned (T7-T10) nanorods with aspect ratios of $\sim 2.5$ and $\sim 3$ and widths from $\sim 7$ to $\sim 16.5 \mathrm{~nm}$ are further probed by the combined TTM-MD approach, and compared with the FE predictions in Fig. 5. Overall, the FE results are in a good match with the periods by the atomistic simulations (within $\sim 5 \%$ ), showing that the adopted continuum model based on the 

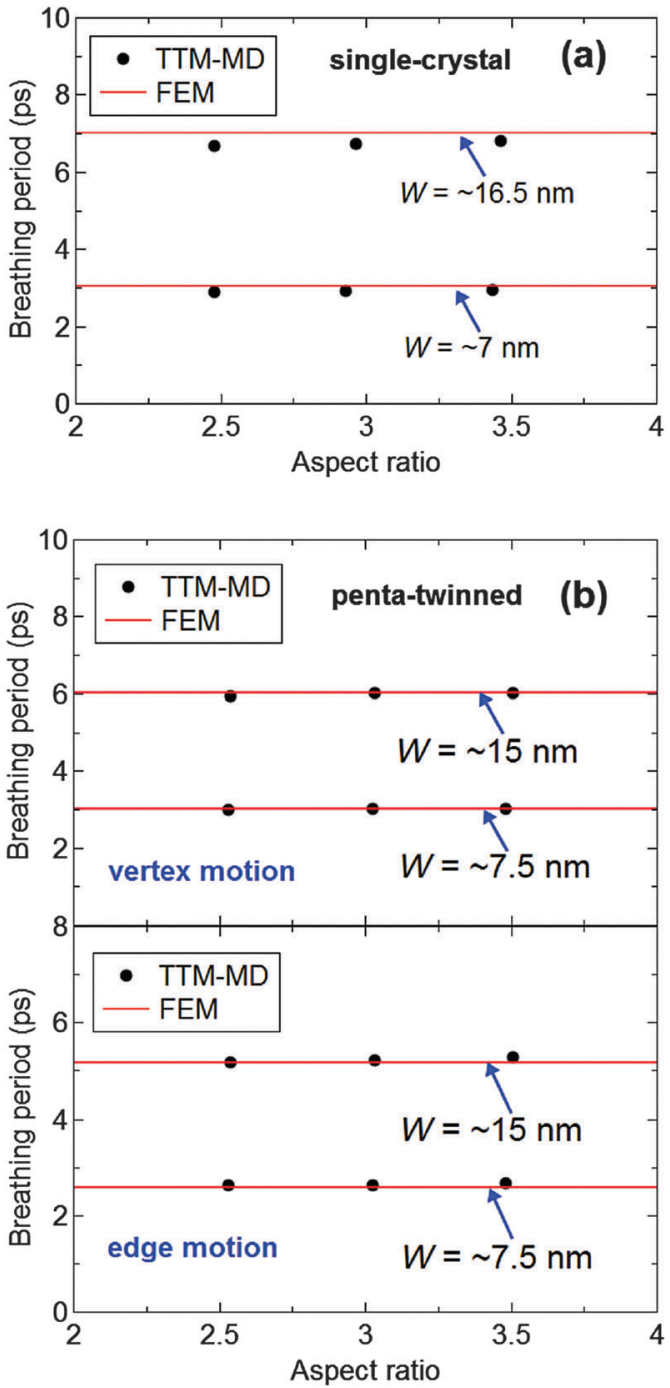

Fig. 5 Comparisons of the breathing periods of gold nanorods with different aspect ratios by the FE calculations and the coupled TTM-MD simulations: (a) single-crystal nanorods and (b) penta-twinned nanorods.

vibrations of an infinitely long rod could be used for gold nanorods with an aspect ratio down to $\sim 2.5$.

It has been reported that there is a trivial crystal structure effect on the breathing mode of metal nanospheres. ${ }^{47}$ It is interesting to know whether the breathing periods of gold nanorods are also little influenced by the crystal structure. In light of the relations between polycrystalline and monocrystalline elastic constants, ${ }^{48}$ the Young's modulus $E=78.74 \mathrm{GPa}$ and Poisson's ratio $\nu=0.43$ for isotropic bulk gold are derived from $c_{11}, c_{12}$, and $c_{44}$ for bulk fcc-crystal gold as obtained by the interatomic EAM potential used above. The breathing periods of samples S1-S5 and T1-T6 via the FE calculations with isotropic $E$ and $\nu$ are also presented in Fig. $2 \mathrm{~d}$ and $3 \mathrm{~d}$.

As can be found from the above figures, the use of isotropic elastic constants does not significantly affect the FE predictions of the breathing periods of the single-crystal nanorods $(\sim 1.6 \%$ difference), suggesting a small crystal structure effect on the breathing mode of the single-crystal nanorods. In comparison,
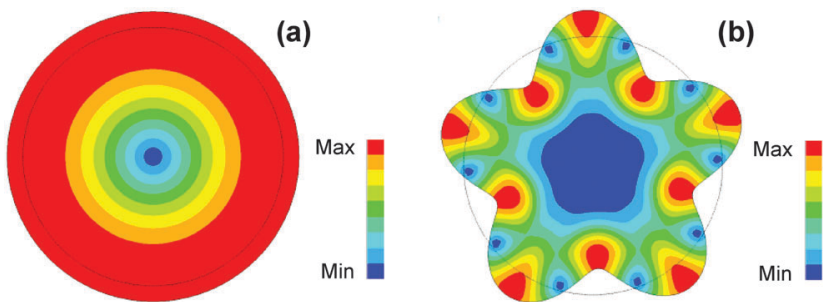

Fig. 6 (a) Fundamental breathing mode and (b) the five-fold surface mode of an isotropic elastic cylinder. The isotropic Young's modulus $E=78.74 \mathrm{GPa}$ and Poisson's ratio $\nu=0.43$ obtained by the EAM potential are used in the FE calculations.

for twinned nanorods, there is some discrepancy between the breathing periods obtained via FE computations with the anisotropic and isotropic bulk elastic constants. Specifically, the differences are $\sim 7 \%$ and $\sim 5 \%$ for the modes with the motion at the vertices and edges of the pentagonal cross-section, respectively. The breathing periods of nanorods depend on the average speed of sound in the crystal directions perpendicular to the long axis of nanorods. ${ }^{21}$ The penta-twinned gold nanorods are composed of five single-crystal triangular prisms stacked together along a common [110] axis (see Fig. 1a). As compared with single-crystal gold nanorods, penta-twinned nanorods contain a smaller range of crystal directions perpendicular to the long axis, causing a larger deviation of the crystal direction averaged speed of sound from the speed of sound in isotropic polycrystalline gold. Hence, the breathing vibrations of twinned gold nanorods are more influenced by the crystal structure effect than those of the singlecrystal nanorods, and the use of isotropic elastic constants is less favorable for twinned gold nanorods.

Finally, we studied the vibrations of infinitely long isotropic elastic cylinders with the same radii as those corresponding to the inscribed circles of the pentagonal cross-sections of nanorods T1-T6. Fig. 6 illustrates the fundamental breathing mode and the five-fold surface mode of an isotropic cylinder, which are similar to the breathing modes of twinned gold nanorods with the edge and vertex motion, respectively. In addition, their periods are found close to those for the breathing modes of the twinned gold nanorods determined by the coupled TTM-MD simulations (less than 5\% error), as shown in Fig. 3d. This confirms the claim by Major et $a .^{46}$ that the two breathing modes of penta-twinned gold nanorods arise from the mixture between the breathing mode of the circular cylinder and the five-fold "whispering-gallery" like surface mode.

\section{Conclusions}

Ultrafast laser-induced vibrations of individual single-crystal and penta-twinned gold nanorods have been investigated using the continuum and atomistic methods. It is clearly demonstrated that the continuum model based on the vibrations of rods with an infinite length is able to predict well the breathing periods of gold nanorods with a width as small as $\sim 7 \mathrm{~nm}$ and an aspect ratio down to $\sim 2.5$, together with the bulk elastic constants. The Young's moduli of gold nanorods are derived via 
their atomistically simulated extensional periods and the dispersion relation by the long-wavelength approximation. The elastic modulus of the twinned gold nanorods increases with decreasing width, showing a size effect opposite to that for singlecrystal nanorods. In addition, the FE calculations with isotropic and anisotropic elastic constants indicate that the breathing vibrations of the twinned nanorods are more affected by the crystal structure effect than those of the single-crystal nanorods, because less crystal directions perpendicular to the long axis of twinned nanorods are involved in their breathing modes.

The present study provides new insights into the breathing vibrations and mechanical properties of single gold nanorods with different sizes and crystal structures, and demonstrates the coupled TTM-MD method as a robust complement to the current experimental approaches for investigating the vibrations of small metallic nanostructures. It is suggested that future efforts utilizing a similar methodology be made to explore the acoustic vibrations of gold nanorods in liquids. The effects of nanoscale solid-fluid interactions on the vibrational period and damping of nanorods and on the non-Newtonian viscoelastic behaviors of high-viscosity liquids would be of particular interest. ${ }^{22-24,49}$

\section{Acknowledgements}

This work was supported by the National Natural Science Foundation of China under Grant No. 11102185. The atomic snapshots in Fig. 2 and 3 are created using the open-source graphics package OVITO. ${ }^{50}$

\section{Notes and references}

1 C. A. Foss, G. L. Hornyak, J. A. Stockert and C. R. Martin, J. Phys. Chem., 1992, 96, 7497-7499.

2 C. A. Foss, G. L. Hornyak, J. A. Stockert and C. R. Martin, J. Phys. Chem., 1994, 98, 2963-2971.

3 N. R. Jana, L. Gearheart and C. J. Murphy, Adv. Mater., 2001, 13, 1389-1393.

4 B. Nikoobakht and M. A. El-Sayed, Chem. Mater., 2003, 15, 1957-1962.

5 X. Huang and M. A. El-Sayed, Adv. Mater., 2009, 21, 4880-4910.

6 C. J. Murphy, T. K. Sau, A. M. Gole, C. J. Orendorff, J. Gao, L. Gou, S. E. Hunyadi and T. J. Li, J. Phys. Chem. B, 2005, 109, 13857-13870.

7 J. Kumar and K. G. Thomas, J. Phys. Chem. Lett., 2011, 2, 610-615.

8 G. V. Hartland, Annu. Rev. Phys. Chem., 2006, 57, 403-430.

9 A. L. Tchebotareva, P. V. Ruijgrok, P. Zijlstra and M. Orrit, Laser Photonics Rev., 2010, 4, 581-597.

10 A. Crut, P. Maioli, N. Del Fatti and F. Vallée, Phys. Rep., 2015, 549, 1-43.

11 Y. T. Yang, C. Callegari, X. L. Feng, K. L. Ekinci and M. L. Roukes, Nano Lett., 2006, 6, 583-586.

12 H. Staleva and G. V. Hartland, Adv. Funct. Mater., 2008, 18, 3809-3817.
13 K. O’Brien, N. D. Lanzillotti-Kimura, J. Rho, H. Suchowski, X. Yin and X. Zhang, Nat. Commun., 2014, 5, 4042.

14 T. A. Major, S. S. Lo, K. Yu and G. V. Hartland, J. Phys. Chem. Lett., 2014, 5, 866-874.

15 A. Crut, P. Maioli, N. Del Fatti and F. Vallée, Ultrasonics, 2015, 56, 98-108.

16 G. V. Hartland, Chem. Rev., 2011, 111, 3858-3887.

17 M. Hu, P. Hillyard, G. V. Hartland, T. Kosel, J. J. Perez and P. Mulvaney, Nano Lett., 2004, 4, 2493-2497.

18 M. Hu, X. Wang, G. V. Hartland, P. Mulvaney, J. J. Perez and J. E. Sader, J. Am. Chem. Soc., 2003, 125, 14925-14933.

19 H. Petrova, J. J. Perez, Z. Y. Zhang, J. Zhang, T. Kosel and G. V. Hartland, J. Mater. Chem., 2006, 16, 3957-3963.

20 M. A. Mahmoud, D. O'Neil and M. A. El-Sayed, Nano Lett., 2014, 14, 743-748.

21 P. Zijlstra, A. L. Tchebotareva, J. W. M. Chon, M. Gu and M. Orrit, Nano Lett., 2008, 8, 3493-3497.

22 P. V. Ruijgrok, P. Zijlstra, A. L. Tchebotareva and M. Orrit, Nano Lett., 2012, 12, 1063-1069.

23 K. Yu, P. Zijlstra, J. E. Sader, Q. H. Xu and M. Orrit, Nano Lett., 2013, 13, 2710-2716.

24 K. Yu, T. A. Major, D. Chakraborty, M. S. Devadas, J. E. Sader and G. V. Hartland, Nano Lett., 2015, 15, 3964-3970.

25 Y. Gan, C. Q. Wang and Z. Chen, Opt. Lett., 2015, 40, 340-343.

26 Y. Gan, Z. Sun and Z. Chen, J. Appl. Phys., 2015, 118, 164304.

27 D. S. Ivanov and L. V. Zhigilei, Phys. Rev. B: Condens. Matter Mater. Phys., 2003, 68, 064114.

28 N. Wang, S. I. Rokhlin and D. F. Farson, J. Nanopart. Res., 2011, 13, 4491.

29 Y. Gan and S. Jiang, J. Appl. Phys., 2013, 113, 073507.

30 S. I. Anisimov, B. L. Kapeliovich and T. L. Perelman, J. Exp. Theor. Phys., 1974, 39, 375-377.

31 C. J. Johnson, E. Dujardin, S. A. Davis, C. J. Murphy and S. J. Mann, Mater. Chem., 2002, 12, 1765-1770.

32 S.-H. Zhang, Z.-Y. Jiang, Z.-X. Xie, X. Xu, R.-B. Huang and L.-S. Zheng, J. Phys. Chem. B, 2005, 109, 9416-9421.

33 Z. L. Wang, M. B. Mohamed, S. Link and M. A. El-Sayed, Surf. Sci., 1999, 440, L809-L814.

34 H. W. Sheng, M. J. Kramer, A. Cadien, T. Fujita and M. W. Chen, Phys. Rev. B: Condens. Matter Mater. Phys., 2011, 83, 134118.

35 Y. Zhen and C. Chu, Comput. Phys. Commun., 2012, 183, 261-265.

36 J. R. Neighbovrs and G. A. Alers, Phys. Rev., 1958, 111, 707-712.

37 Z. Lin, L. V. Zhigilei and V. Celli, Phys. Rev. B: Condens. Matter Mater. Phys., 2008, 77, 075133.

38 S. Nosé, J. Chem. Phys., 1984, 81, 511-519.

39 W. G. Hoover, Phys. Rev. A: At., Mol., Opt. Phys., 1985, 31, 1695-1697.

40 M. Allen and D. Tildesley, Computer Simulation of Liquids, Oxford University Press, New York, 1987.

41 A. F. Bower, Applied Mechanics of Solids, CRC Press, Boca Raton, FL, 2010.

42 A. E. H. Love, A Treatise on the Mathematical Theory of Elasticity, Dover Publications, New York, 4th edn, 1944. 
43 F. Niekiel, E. Spiecker and E. Bitzek, J. Mech. Phys. Solids, 2015, 84, 358-379.

44 H. Liang, M. Upmanyu and H. Huang, Phys. Rev. B: Condens. Matter Mater. Phys., 2005, 71, 241403(R).

45 Z.-J. Wang, C. Liu, Z. Li and T.-Y. Zhang, J. Appl. Phys., 2010, 108, 083506.

46 T. A. Major, A. Crut, B. Gao, S. S. Lo, N. Del Fatti, F. Vallée and G. V. Hartland, Phys. Chem. Chem. Phys., 2013, 15, 4169-4176.
47 V. Juvé, A. Crut, P. Maioli, M. Pellarin, M. Broyer, N. Del Fatti and F. Vallée, Nano Lett., 2010, 10, 1853-1858.

48 A. Crut, P. Maioli, N. Del Fatti and F. Vallée, Phys. Chem. Chem. Phys., 2009, 11, 5882-5888.

49 M. Pelton, D. Chakraborty, E. Malachosky, P. Guyot-Sionnest and J. E. Sader, Phys. Rev. Lett., 2013, 111, 244502.

50 A. Stukowski, Modell. Simul. Mater. Sci. Eng., 2010, 18, 015012. 